Article

\title{
Multi-Beam Circular Polarized Reflectarray on Parabolic Reflector by Variable Rotation Technique
}

\author{
Álvaro Somolinos ${ }^{1}\left(\mathbb{D}\right.$, Rafael Florencio ${ }^{2, *}$, Iván González ${ }^{2}$ and Felipe Cátedra ${ }^{2}$ \\ 1 newFASANT, 19005 Guadalajara, Spain \\ 2 Department of Computer Science, University of Alcalá, 28805 Alcalá de Henares, Spain \\ * Correspondence: rafaelflorenciodiaz@yahoo.es
}

Received: 20 May 2019; Accepted: 28 June 2019; Published: 29 June 2019

check for updates

\begin{abstract}
Multi-beams antennas are currently being used for direct broadcast satellite, personal communication satellite, military communication satellite, and high-speed internet applications. In this work, a circularly polarized $(\mathrm{CP})$ multi-spot beam satellite parabolic reflectarray antenna is designed to provide six spot beams at $19.7 \mathrm{GHz}$. For this purpose, an easy technique to compute the required phase shifts to produce two focused beams in specular directions for a $\mathrm{CP}$ parabolic reflectarray is proposed. These required phase shifts are added to the reflected fields by the variable rotation of the reflectarray elements printed on the surface of a parabolic antenna which are fed by a dual-CP feed-horn. For this purpose, a reflectarray cell made of a conductive cross embedded in a grounded multilayered substrate is optimized to produce very linear phase-shift and low cross-polarization level. To demonstrate the multibeam capacity, a 1.8-meter offset parabolic reflectarray made of the optimized reflectarray element was designed to generate six focused beams in dual-CP with three dual-CP feed-horns. The six main spots fulfill the typical multi spot satellite requirement with angular separation less than $0.56^{\circ}, 0.4 \mathrm{~dB}$ loss in the gain, and cross-polarization level below $35 \mathrm{~dB}$ with respect to the maximum of radiation.
\end{abstract}

Keywords: reflectarray; satellite antennas; multibeam antennas; moment method

\section{Introduction}

The development of wireless communication systems has been widely demanded for many application areas, such as in car-to-car communications, car-to-direct-broadcast-satellite communications, and car-to-roadside-unit communications. For antennas in car-to-car communications applications, the use of several radiators is a prominent solution. However, placing several radiators within the small space of a vehicle is a daunting task [1]. Therefore, multiband [2,3] and/or wideband antennas [4-6] of small size which provide broad beamwidth are desired. These properties make antennas form reliable links during the vehicle's mobility. For car-to-direct-broadcast-satellite communications, it is desirable to have high gain and circularly polarized (CP) antennas with wide beam scanning angles [7-11]. The wide beam scanning angles enable vehicles to keep track of the satellites so that they are always within the reception area of the satellite and do not fall within blind spots [9]. In the case of car-to-roadside-unit communications, the standard specifications require the uses of antennas with radiation patterns that provide roughly a half-power beamwidth of $20^{\circ}$ to confine the main lobe and sidelobe level $15 \mathrm{~dB}$ lower than those of the boresight [12].

In other important application areas, such as direct broadcast satellite service, military communication satellite, and high-speed internet applications, the antenna system is hosted on the satellite. Multi-spot beam satellite antennas are promising to offer contiguous coverage over a specified field of view on Earth for dual-polarization transmit-receive applications $[13,14]$. In order to avoid mutual interference between the spot beams, a four-color frequency and polarization reuse 
scheme is usually employed $[13,14]$. In this scheme, two different frequency sub-bands and two orthogonal polarizations are used such that each spot in two adjacent colors could differ in frequency, polarization, or both [15]. In a four-color configuration, the antenna system shown in $[13,14]$ employs one reflector aperture per color. Although there are system configurations which reduce the profile of four reflector antennas by means of linear or circular polarization selective surfaces [16], the mass reflector antennas and complex mechanical systems are unsuitable for satellites. Technology based on printed reflectarrays offers a reduction in the number of apertures in the satellite, keeping the stringent requirements of frequency band and cross-polarizations [17,18].

Reflectarray elements with variable rotation are promising for the generation of a pencil beam in circular polarization with very low levels of cross-polarization [19]. In fact, in [19] it is shown that phase shifts with opposite signs are added to the co-polar components of the $\mathrm{CP}$ reflected fields when the co-polar reflection coefficients of the two orthogonal linear polarizations are of equal magnitude and opposite sign. These opposite phase shifts depend on twice the rotation angle of the rotating element. This is known as the variable rotation technique (VRT). In [15], these properties was exploited to tilt the dual CP focused beams in specular directions.

In this work, we present analysis and design techniques of multi-beam parabolic reflectarrays in dual circular polarization exploiting properties associated with the variable rotation of reflectarray elements, similar to the work presented in [15] but with some important differences. First, we explore the usage of rotating elements that by themselves provide the reflection properties required by the VRT. In particular, we chose a conductive cross as the rotating element. Contrary to the single dipoles proposed in [15], these elements depend weakly on the thickness and other parameters of the reflectarray dielectric layers. Moreover, the conductive cross roughly offers the possibility of independent phase control on orthogonal field components for linear polarization. This independent phase control provides more flexibility in the design process in order to correct phase errors. For example, a small phase correction can be added on both polarizations to improve the beam shaping. Second, the reflectarray cells are full-wave analyzed under local periodicity assumption [20] using a method of moments (MoM) formulation in the "space domain" for planar periodic multilayer structures [21], which provides a great flexibility for the fast and accurate design of unit cells with arbitrarily shaped elements. Third, in spite of using a fast and accurate tool for the analysis of periodic multilayer structure [21], we propose an easy strategy for the optimization and interpolation of the geometric parameters of the reflectarray element to provide few calls to full-wave analysis routine in the design process-this will provide reduction in the consumption of the CPU time in the design process. Fourth, although in this work a specific design is carried out on a parabolic surface, the proposed scheme for designing multi-beam antennas using the VRT is shown on an arbitrarily shaped surface.

\section{Required Phase Shift to Tilt Dual CP Focused Beams in Specular Directions}

Figure 1 shows the side view of a reflectarray on an arbitrary reflector surface with the phase center of the feed at the source point. In this section, we present an easy technique for computing the required phase distribution to be added in reflected waves by the rotating elements in order to tilt dual $\mathrm{CP}$ focused beams in specular directions. Ray optics theory is considered in this formulation. 


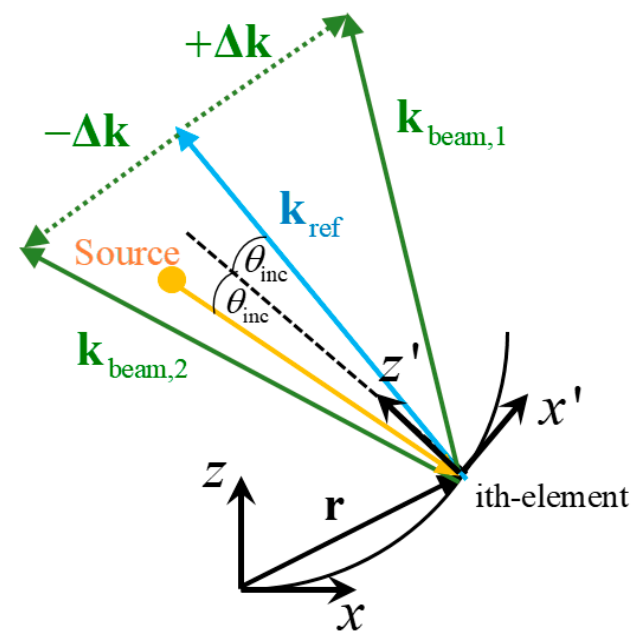

Figure 1. Scheme of a reflectarray on an arbitrary surface reflector. The curved black solid line indicates the arbitrary surface (flat or curved) of the reflectarray. The propagation vectors of the impinging and reflected waves by law of reflection are shown. The propagation vectors of the desired reflected waves are also shown. These last vectors are specular vectors with respect to the direction of the propagation vector $\mathbf{k}_{\text {ref. }}$.

Let us consider the impinging wave on the $i$-th element of the reflectarray with propagation vector given by $\mathbf{k}_{\text {inc }}=k_{0}\left[\sin \left(\theta_{\text {inc }}\right) \cos \left(\phi_{\text {inc }}\right) \hat{\mathbf{x}}^{\prime}+\sin \left(\theta_{\text {inc }}\right) \sin \left(\phi_{\text {inc }}\right) \hat{\mathbf{y}}^{\prime}-\cos \left(\theta_{\text {inc }}\right) \hat{\mathbf{z}}^{\prime}\right]$ where $k_{0}$ is the vacuum wavenumber at the working frequency and $\theta_{\text {inc }}, \phi_{\text {inc }}$ are the spherical angular coordinates of the impinging wave in the local reference system on the $i$-th element (see $x^{\prime}-z^{\prime}$ axis in Figure 1). According to the law of reflection, a reflected wave will be produced with the propagation vector $\mathbf{k}_{\text {ref }}=k_{0}\left[\sin \left(\theta_{\text {inc }}\right) \cos \left(\phi_{\text {inc }}\right) \hat{\mathbf{x}}^{\prime}+\sin \left(\theta_{\text {inc }}\right) \sin \left(\phi_{\text {inc }}\right) \hat{\mathbf{y}}^{\prime}+\cos \left(\theta_{\text {inc }}\right) \hat{\mathbf{z}}^{\prime}\right]$. In this way, the phase of the reflected wave will be $\mathbf{k}_{\text {ref }} \cdot \mathbf{r}$, where $\mathbf{r}$ is the vector position of the $i$-th element of the reflectarray with respect to the global reference system shown in Figure 1 (see the $x-z$ axis in Figure 1). Now, let us consider a desired reflected wave with propagation vector given by $\mathbf{k}_{\text {beam }, 1}=k_{0}\left[\sin \left(\theta_{\text {beam }, 1}\right) \cos \left(\phi_{\text {beam }, 1}\right) \hat{\mathbf{x}}^{\prime}+\sin \left(\theta_{\text {beam }, 1}\right) \sin \left(\phi_{\text {beam }, 1}\right) \hat{\mathbf{y}}^{\prime}+\cos \left(\theta_{\text {beam }, 1}\right) \hat{\mathbf{z}}^{\prime}\right]$, where $\theta_{\text {beam }, 1}$ and $\phi_{\text {beam, } 1}$ are the spherical angular coordinates of the desired beam direction in the local reference system on the $i$-th element (see again the $x^{\prime}-z^{\prime}$ axis in Figure 1). In order to obtain the desired reflected wave with the desired beam direction, the $i$-th element should introduce to the reflected wave the additional phase given by:

$$
\Delta \mathbf{k} \cdot \mathbf{r}=\left(\mathbf{k}_{\text {beam }, 1}-\mathbf{k}_{\text {ref }}\right) \cdot \mathbf{r} .
$$

On the other hand, let us consider a reflected wave with propagation vector $\mathbf{k}_{\mathbf{b e a m}, 2}=$ $k_{0}\left[\sin \left(\theta_{\text {beam }, 2}\right) \cos \left(\phi_{\text {beam, } 2}\right) \hat{\mathbf{x}}^{\prime}+\sin \left(\theta_{\text {beam,2 }}\right) \sin \left(\phi_{\text {beam }, 2}\right) \hat{\mathbf{y}}^{\prime}+\cos \left(\theta_{\text {beam }, 2}\right) \hat{\mathbf{z}}^{\prime}\right]$ (Figure 1$)$, where $\theta_{\text {beam,2 }}$ and $\phi_{\text {beam, }, 2}$ are the spherical angular coordinates of the specular beam direction in the local reference system on the $i$-th element (see again the $x^{\prime}-z^{\prime}$ axis in Figure 1 ). According to Figure 1, this direction of the reflected wave is the specular direction of the direction given by $\mathbf{k}_{\text {beam, } 1}$ with respect to the propagation vector $\mathbf{k}_{\text {ref. }}$. In order to obtain this reflected wave with this specular beam direction, the $i$-th element should introduce to the reflected wave the additional phase given by:

$$
-\Delta \mathbf{k} \cdot \mathbf{r}=\left(\mathbf{k}_{\text {ref }}-\mathbf{k}_{\text {beam }, 1}\right) .
$$

The additional phases given in (1) and (2) are of equal magnitude and opposite sign. These opposite additional phases can be added to the phase of the reflected wave using the same layout by VRT [15]. Note that the additional phases given in (1) and (2) can be computed by an arbitrary surface (i.e., the vector position $\mathbf{r}$ does not suffer any constraint). In fact, the proof of the concept is demonstrated experimentally in [22] when the arbitrary surface is a flat surface for non-focused beams in CP. In this work, we considered the particular case of a parabolic surface to apply the VRT. 
The parabolic surface focuses the beams in CP while the VRT is applied to introduce the additional phases given in (1) and (2) to the phase of the reflected waves to produce two focused beams in specular directions.

We consider an offset parabolic reflector shown in Figure 2 with parabola vertex at the coordinate origin. The remaining geometric parameters are the following: focus in the $z$-axis at $2.718 \mathrm{~m}$ height, symmetry plane of the parabola at $y=0$, diameter of the disk of $1.812 \mathrm{~m}$, clearance of $0.35 \mathrm{~m}$ (focal length/diameter ratio is 1.5). The $\mathbf{k}_{\text {ref }}$ direction of the main beam without introducing the opposite additional phases is the $z$-axis. The specular desired directions were $\theta_{\text {beam }, 1}=+0.28 \mathrm{deg}$, $\phi_{\text {beam }, 1}=0 \mathrm{deg}$ and $\theta_{\text {beam }, 2}=-0.28 \mathrm{deg}, \phi_{\text {beam }, 2}=0 \mathrm{deg}$ of the main beams.

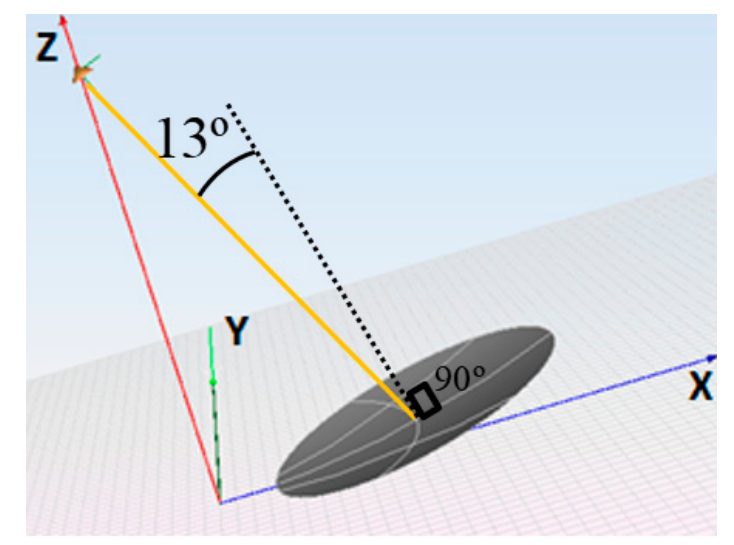

Figure 2. Scheme of offset parabolic reflector. The feed location and incident angle of the central element are shown.

The incident angle $\theta_{\text {inc }}$ which suffers the central element of the parabolic reflectarray is $13.03 \mathrm{deg}$. Note that for most elements of the parabolic reflectarray, the incident angle $\theta_{\text {inc }}$ can be approximated by its value at the center of the parabolic reflectarray. This will be exploited in the next section in order to optimize the reflectarray element to provide few calls to the full-wave analysis routine in the design process to reduce the consumption of CPU time in the design process.

For this reflector, the maximum and minimum values of the additional phase given by (1) were obtained for the extreme values of $x$ coordinates, which were $0.35 \mathrm{~m}$ and $2.162 \mathrm{~m}$, respectively. The difference of the values that takes in the extreme values for $\theta_{\text {beam }, 1}=+0.28 \mathrm{deg}$ was 3.66 radians at 19.7 GHz. If we consider the value of the additional phase given by (1) for the center of the disk, we note that the phase change was only 1.83 radians from this center to any extreme point of the disk (i.e., only $105 \mathrm{deg}$ ). According to $[15,19]$ there is a rule stating that the phase shift added by the rotating element is twice the rotation angle. This rule is quite accurate when the elements are much smaller than the operation wavelength. In that case, the coupling between elements should not change much with rotation. However, as shown in the next section, the proposed reflectarray element has a period size roughly half of the operation wavelength and the variation of the coupling with rotation is not negligible. So, using the rule that the phase shift added by the rotating element is twice the rotation angle, we can state that regarding the angular rotation value at the center of the disk, the unit element rotation angle is always in the range from -52.5 to +52.5 degrees.

\section{Design and Optimization of The Rotating Element of the Reflectarray}

We consider a left-handed circular polarized (LHCP) impinging plane wave on a multilayer periodic structure with the unit cell shown in Figure 3. This unit cell is made of two dielectric layers. The bottom $2 \mathrm{~mm}$ dielectric layer comprised of Rohacell material is used as separator with the ground plane. The upper $0.127 \mathrm{~mm}$ dielectric layer comprised of Arlon material is used to host a conductive cross printed on its lower limit as shown in Figure $3 \mathrm{~b}$. The reflectarray cells have a quasi-periodicity, with a period in $\mathrm{mm}$ of $7.6 \times 7.6(0.499 \times 0.499$ in wavelengths). This reflectarray element was used 
in [22] in order to demonstrate experimentally the proof of the concept of generating two beams in specular directions for $\mathrm{CP}$, and satisfactory results were obtained.

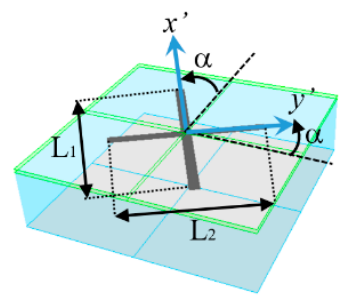

(a)

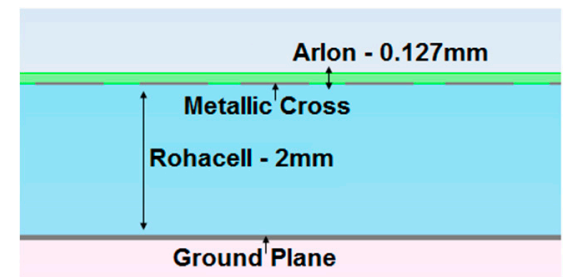

(b)

Figure 3. (a) Definition of the unit cell. The period is $7.6 \mathrm{~mm}$. The components $x^{\prime}$ and $y^{\prime}$ of the incident field are parallel to the larger and shorter cross arms of the figure. (b) Side view of the periodic multilayer structure. The bottom dielectric layer is made of Rohacell material (permittivity $\varepsilon_{r}=1.12$ and loss $\operatorname{tangent} \tan \delta=0.002$ ) and the upper layer is made of Arlon material (permittivity $\varepsilon_{r}=2.3$ and loss tangent $\tan \delta=0.002$ ).

The direction of the impinging plane wave is the $-\hat{\mathbf{z}}^{\prime}$ direction and its electric field is expressed as:

$$
\mathbf{E}_{\text {inc }}^{\mathrm{LHCP}}=E_{0}\left(\hat{\mathbf{x}}^{\prime}-j \hat{\mathbf{y}}^{\prime}\right) e^{j k_{0} z},
$$

where $\hat{\mathbf{x}}^{\prime}$ and $\hat{\mathbf{y}}^{\prime}$ are the unitary vector parallel to the $x^{\prime}$ and $y^{\prime}$ axes as shown in Figure 3 a. These unitary vectors are parallel to the larger and shorter cross arms of Figure 3a. According to [19] and [23], when the LHCP wave is incident on the periodic structure, the electric field of the reflected wave has two contributions of circularly polarized waves: a co-polar contribution given by an LHCP wave and a cross-polarization contribution given by a right-handed circularly polarized (RHCP) wave. Both circularly polarized waves propagate in the positive direction $+\hat{\mathbf{z}}^{\prime}$,

$$
\begin{aligned}
\mathbf{E}_{\mathrm{ref}} & =\frac{1}{2} e^{-j 2 \alpha} E_{0}\left(R_{x^{\prime} x^{\prime}}-R_{y^{\prime} y^{\prime}}\right)\left(\hat{\mathbf{x}}^{\prime}+j \hat{\mathbf{y}}^{\prime}\right) e^{-j k_{0} z^{\prime}} \\
& +\frac{1}{2} E_{0}\left(R_{x^{\prime} x^{\prime}}+R_{y^{\prime} y^{\prime}}\right)\left(\hat{\mathbf{x}}^{\prime}-j \hat{\mathbf{y}}^{\prime}\right) e^{-j k_{0} z \prime}
\end{aligned}
$$

where $R_{x^{\prime} x^{\prime}}$ and $R_{y^{\prime} y^{\prime}}$ are the reflection coefficients for the co-polar components in directions of the $x^{\prime}$ and $y^{\prime}$ axes, respectively, as shown in Figure 3a (i.e., in the directions of the crossed arms) and $\alpha$ is the angle of rotation of the crosses shown in Figure 3a. In (4) it is assumed that cross-polarization reflection coefficients $R_{x^{\prime} y^{\prime}}$ and $R_{y^{\prime} x^{\prime}}$ are negligible. This assumption is based on the symmetry of the reflectarray element. For example, let us consider an impinging plane wave with its electric field linearly polarized in $x^{\prime}$. In the cross arm oriented in the $x^{\prime}$ direction, due to the symmetry of the arm regarding the $x^{\prime}$ axis, there is no reason to expect a contribution to the reflected electric field with $y^{\prime}$ polarization. The same rationale can be applied for the cross arm oriented in the $y^{\prime}$ direction. As will be shown later, this assumption leads to an equation which predicts the level of the cross-polarization contribution of a circularly polarized wave with enough accuracy for practical purposes. Note that the first contribution in (4) is the co-polar reflected electric field of an LHCP wave, since the impinging waves propagate in the $-\hat{\mathbf{z}}^{\prime}$ direction and the reflected wave propagates in $+\hat{\mathbf{z}}^{\prime}$ direction. The second contribution in (4) is the cross-polarization reflected electric field of an RHCP wave. According to (4), when $R_{x^{\prime} x^{\prime}}=-R_{y^{\prime} y^{\prime}}$, a pure reflected LHCP wave is obtained (there is no cross-polarization contribution). Moreover, the added phase shift by the rotating element to the reflected electric field of this pure LHCP wave is $-2 \alpha$. Indeed, when $R_{x^{\prime} x^{\prime}}=-R_{y^{\prime} y^{\prime}}$, the reflected field is:

$$
\mathbf{E}_{\text {ref }}^{\mathrm{LHCP}}=e^{j\left(-2 \alpha+\angle R_{x^{\prime} x^{\prime}}\right)}\left|R_{x^{\prime} x^{\prime}}\right| E_{0}\left(\hat{\mathbf{x}}^{\prime}+j \hat{\mathbf{y}}^{\prime}\right) e^{-j k_{0} z^{\prime}} .
$$


Note that when there is no rotation, the phase shift of the reflected electric field of the pure LHCP wave is equal to the phase of the $R_{x^{\prime} x^{\prime}}$ reflection coefficient. The phase of $R_{x^{\prime} x^{\prime}}$ offers an additional degree of freedom to modify the phase of the reflected wave in (5). This additional degree of freedom can be exploited by means of the use of conductive cross. In the case of conductive cross, roughly independent phase control of reflection coefficients $R_{x^{\prime} x^{\prime}}$ and $R_{y^{\prime} y^{\prime}}$ is possible from independent variations of the lengths $\mathrm{L}_{1}$ and $\mathrm{L}_{2}$. This independent phase control provides more flexibility in the design process since the condition $R_{x^{\prime} x^{\prime}}=-R_{y^{\prime} y^{\prime}}$ can be satisfied for several values of the phase of $R_{x^{\prime} x^{\prime}}$. These several values of the phase of $R_{x^{\prime} x^{\prime}}$ can be used in order to correct phase errors. For example, a small phase correction can be added on both polarizations to improve the beam shaping.

In a similar way, when the impinging wave is RHCP, and $R_{x^{\prime} x^{\prime}}=-R_{y^{\prime} y^{\prime}}$, the reflected electric field of the contribution of the RHCP wave will suffer a phase shift with opposite sign, $+2 \alpha$, as stated in [15]. When the condition $R_{x^{\prime} x^{\prime}}=-R_{y^{\prime} y^{\prime}}$ is not exactly fulfilled, else $R_{x^{\prime} x^{\prime}}=-R_{y^{\prime} y^{\prime}} e^{j \Delta \varphi}$, where an $\Delta \varphi$ is an error in the phase, then the cross-polarization contribution in (4) will be given by:

$$
\mathbf{E}_{\mathrm{ref}}^{\mathrm{RHCP}}=\frac{1}{2} E_{0} R_{x^{\prime} x^{\prime}}\left(1-e^{j \Delta \varphi}\right)\left(\hat{\mathbf{x}}^{\prime}-j \hat{\mathbf{y}}^{\prime}\right) e^{-j k_{0} z^{\prime}} .
$$

Note that this cross-polarization contribution does not depend on the rotation angle, and only depends of the error in the phase $\Delta \varphi$. So, the amplitude of the cross-polarization contribution increases as this phase error $\Delta \varphi$ increases. Recall that (6) was obtained under the assumption that cross-polarization reflection coefficients $R_{x^{\prime} y^{\prime}}$ and $R_{y^{\prime} x^{\prime}}$ are negligible. This equation predicts the cross-polarization level in the circularly polarized reflected wave.

A parametric study of the rotating element of the unit cell shown in Figure 3 was carried out in order to check the performances of the previously shown co-polar and cross-polarization contributions. This parametric study was carried out with the periodic structure module of the newFASANT suite [21]. In this parametric study, the working frequency was $19.7 \mathrm{GHz}$. Since the incident angle $\theta_{\text {inc }}$ in most elements of the parabolic reflectarray can be approximated by the incident angle at the center of the parabolic reflectarray, the incident angles $\theta_{\text {inc }}=13.03 \mathrm{deg}$ and $\phi_{\mathrm{inc}}=0 \mathrm{deg}$ were considered in the parametric study. Since variations of the lengths $\mathrm{L}_{1}$ and $\mathrm{L}_{2}$ produce roughly independent variations on the phase of $R_{x^{\prime} x^{\prime}}$ and $R_{y^{\prime} y^{\prime}}$, these lengths can be adjusted to comply with the condition $R_{x^{\prime} x^{\prime}}=-R_{y^{\prime} y^{\prime}}$. There are many pairs of lengths $\left(\mathrm{L}_{1}, \mathrm{~L}_{2}\right)$ which provide $180^{\circ}$ phase difference. The selected lengths were $\mathrm{L}_{2}=4.9 \mathrm{~mm}$ and $\mathrm{L}_{1}=6.025 \mathrm{~mm}$. A parametric study of rotating of the cross was performed considering the arms' lengths obtained in the previous adjustment.

Figure 4a shows the phases of the reflected electric fields of the co-polar circularly polarized waves with respect to the rotation angle. The phase for RHCP increased with the rotation angle with an opposite sense to the phase for LHCP, as predicted in (5). The amplitude of the reflected electric field of the cross-polar circularly polarized wave for the RHCP incident wave is shown in Figure $4 \mathrm{~b}$. Similar results were obtained for the LHCP incident wave. In this figure we notice that the amplitude of the reflected electric field of the cross-polar circular polarized wave was only negligible when the rotating angle was about 0,90 , or -90 degrees (i.e., a pure RHCP reflected wave is produced). However, for 45 or -45 degrees it was far from being negligible. 


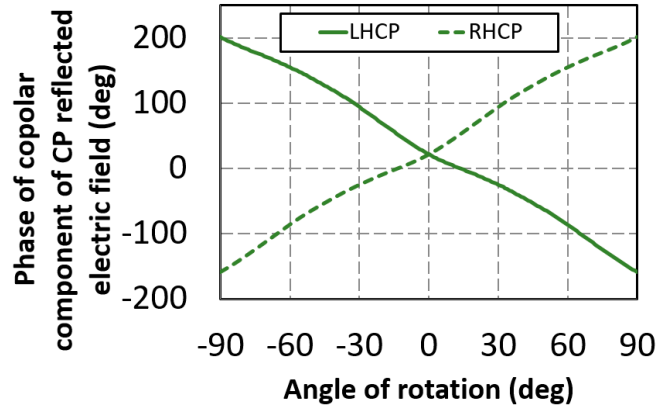

(a)

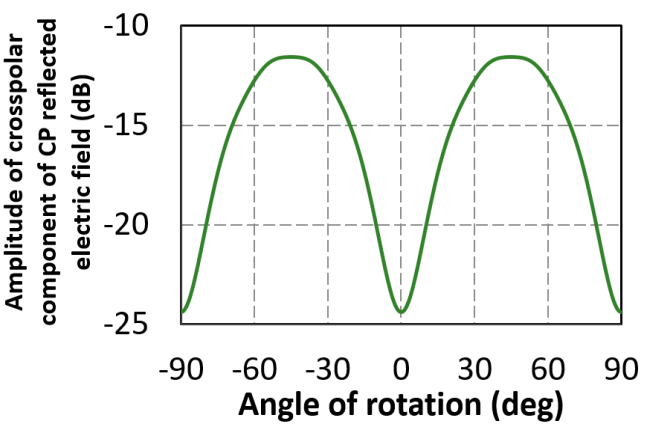

(b)

Figure 4. Electromagnetic response provided by the unit cell at $19.7 \mathrm{GHz}$ with fixed values of $\mathrm{L}_{2}=4.9 \mathrm{~mm}$ and $\mathrm{L}_{1}=6.025 \mathrm{~mm}$ as a function of rotation angle. (a) Phases of the reflected electric fields of the co-polar circularly polarized waves. (b) Amplitude of the reflected electric field of the cross-polar circularly polarized wave for a right-handed circularly polarized (RHCP) incident wave.

This behavior can be explained considering the views of the periodic lattice for two values of the rotational angle: 0 and 45 degrees. We can compare the reflected waves of a linearly polarized incident wave whose components were parallel to the cross arms for rotating angle values of 0 and 45 degrees. If for this case we compare the geometry visualized in Figure $5 \mathrm{a}, \mathrm{b}$ it is obvious that we had two different periodic structures and therefore it could happen that the geometry of Figure $5 \mathrm{c}$ would not give a 180 deg phase shift between the linear co-polar reflection components (i.e., the condition $R_{x^{\prime} x^{\prime}}=-R_{y^{\prime} y^{\prime}}$ is not satisfied), although the geometry was adjusted to give that shift when the rotating angle was $0^{\circ}$ (i.e., the cross was optimized for a $0^{\circ}$ rotation angle but not for $45 \mathrm{deg}$ rotation).

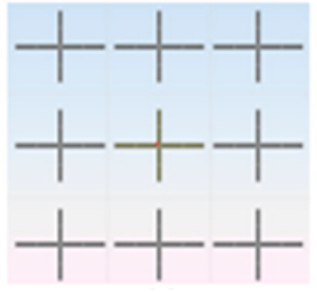

(a)

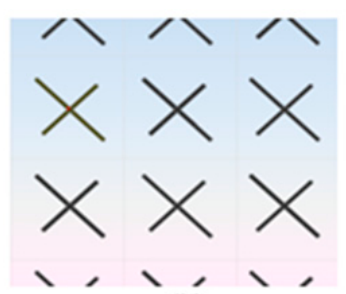

(b)

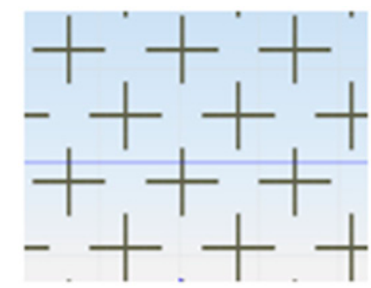

(c)

Figure 5. Aspect of the periodic lattice: (a) cross element on its original position with its arms parallel to the vertical and horizontal lines of the paper; (b) cross element rotated $45 \mathrm{deg}$; (c) cross element rotated $45 \mathrm{deg}$ shown when the paper was also rotated $45 \mathrm{deg}$.

To avoid the issues found in the previous parametric study, a few pairs of lengths $\left(L_{1}, L_{2}\right)$ were optimized in order to fulfill the condition $R_{x^{\prime} x^{\prime}}=-R_{y^{\prime} y^{\prime}}$. The first pair of lengths was optimized when there was no rotation. The obtained values were $\mathrm{L}_{1}=6.0$ and $\mathrm{L}_{2}=4.9 \mathrm{~mm}$. According to previous discussion and Figure 5, the geometry lattice at the rotation angle of 22.5 and 45 degrees differed significantly from the geometry lattice for the non-rotated cross. So, the values of $\mathrm{L}_{1}=6.0$ and $\mathrm{L}_{2}=4.9 \mathrm{~mm}$ would provide significant phase error values $\Delta \varphi$ and similar high cross-polarization level to those shown in Figure $4 \mathrm{~b}$. So, length optimization was imposed at the rotation angles of 22.5 and 45 degrees to avoid the high values of cross-polarization level. In this way, the second and third pairs of lengths were optimized to fulfill the condition $R_{x^{\prime} x^{\prime}}=-R_{y^{\prime} y^{\prime}}$ for rotation angles 22.5 and 45.0 degrees. The obtained values were $\mathrm{L}_{1}=6.14, \mathrm{~L}_{2}=4.91 \mathrm{~mm}$ for the rotation angle of $22.5 \mathrm{deg}$ and $\mathrm{L}_{1}=6.24, \mathrm{~L}_{2}=$ $4.92 \mathrm{~mm}$ for the rotation angle of $45.0 \mathrm{deg}$. We checked that the optimized values of the pair $\left(\mathrm{L}_{1}, \mathrm{~L}_{2}\right)$ for rotation angles of 22.5 and 45.0 degrees also roughly fulfilled the condition $R_{x^{\prime} x^{\prime}}=-R_{y^{\prime} y^{\prime}}$ for rotation angles of -22.5 and -45.0 degrees. Note that two pairs of them provided a 180deg difference of phase for a rotation angle of $45 \mathrm{deg}$ (i.e., for significant change of the periodic lattice). Therefore, we have for the $(-52.5,52.5)$ deg range of the rotation angle samples each $22.5 \mathrm{deg}$ for the lengths of the cross arms 
that were optimized for the VRT. These samples defined six angular intervals in the $(-52.5,52.5) \mathrm{deg}$ rotation range. So, the pairs of lengths were obtained for any rotation angle by linear interpolation in the corresponding angular interval. The interpolation in these six angular intervals would provide few calls to a full-wave analysis routine in the design process of the parabolic reflectarray and the CPU time of the design process would be reduced.

Figure 6a shows the value of the phase error $\Delta \varphi$ produced in the $180^{\circ}$ of difference of phase between the reflection coefficients $R_{x^{\prime} x^{\prime}}$ and $R_{y^{\prime} y^{\prime}}$ at $19.7 \mathrm{GHz}$. These values of phase error are shown as a function of the rotation angle. A maximum phase error of $7^{\circ}$ was obtained. This phase error predicts $-24 \mathrm{~dB}$ of amplitude of the reflected electric field of the cross-polar circularly polarized wave (see (6) with $\mathrm{E}_{0}=1$ ).

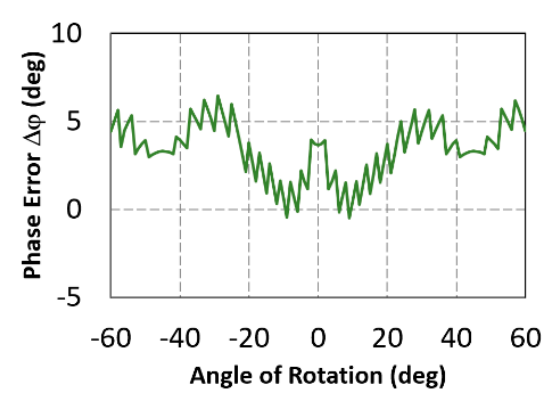

(a)

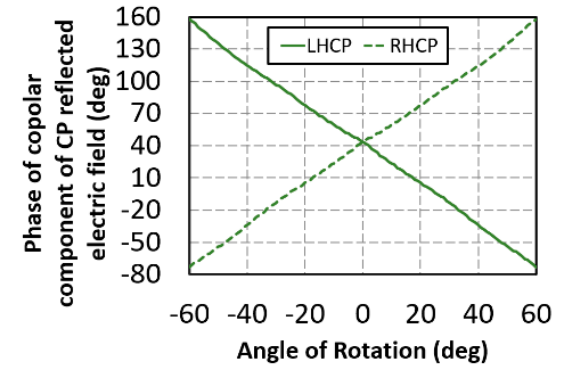

(b)

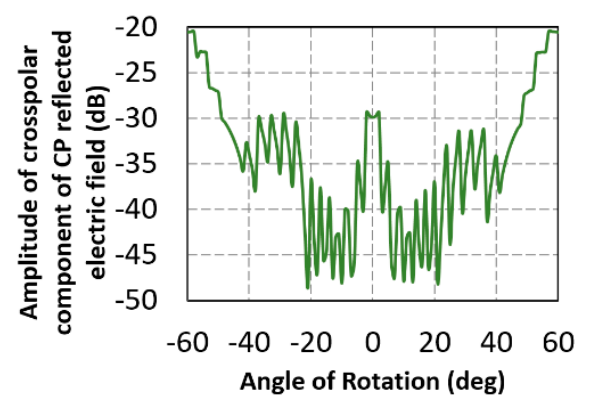

(c)

Figure 6. Electromagnetic response of the optimized cell provided by the unit cell at $19.7 \mathrm{GHz}$ as a function of the rotation angle. (a) Phase error $\Delta \varphi$ produced in the 180deg of difference of phase between the reflection coefficients $R_{x^{\prime} x^{\prime}}$ and $R_{y^{\prime} y^{\prime}}$. (b) Phases of the reflected electric fields of the co-polar circular polarized waves. (c) Amplitude of the reflected electric field of the cross-polar circularly polarized wave for an RHCP incident wave.

Figure $6 \mathrm{~b}$ shows the phase of the reflected electric field of the co-polar circular polarized wave as a function of the rotation angle. Very linear dependence with respect of rotation angle was obtained. These dependences were roughly $+2 \alpha$ for RHCP and $-2 \alpha$ for LHCP reflected waves, as expected from (5). The amplitude of the reflected electric field of the cross-polar circularly polarized wave for RHCP incident wave is shown in Figure 6c. Similar cross-polarization results were obtained for an LHCP incident wave. We see that the cross-polarization level was between -30 and $-20 \mathrm{~dB}$, as predicted from (6).

\section{Optimized Design of a Multibeam Parabolic Reflectarray}

Using the parabolic reflector described in Section 2 as a grounded surface, a parabolic reflectarray was designed using the reflectarray element designed in the previous section. The design was implemented using the VRT to match the required phase shift given by (1) and (2) to tilt dual $\mathrm{CP}$ focused beams in the specular directions given by $\theta_{\text {beam, } 1}=+0.28 \mathrm{deg}, \phi_{\text {beam,1 }}=0 \mathrm{deg}$ and $\theta_{\text {beam }, 2}=-0.28 \mathrm{deg}, \phi_{\text {beam }, 2}=0 \mathrm{deg}$. 
To demonstrate the multi-beam capacity of the designed reflectarray, we simulated the antenna considering three dual CP feed horns: one in the focus and the other two with their $x$-coordinates shifted $\pm 55 \mathrm{~mm}$. We considered the two circular polarizations for each feed position. For each dual $\mathrm{CP}$ feed-horns their $\mathrm{H}$ and $\mathrm{E}$ plane patterns were modeled $\operatorname{as}^{2} \cos ^{24}(\theta)$ [24]. With these positions and polarizations of each feed, we obtained the six main spot beams. Figure $7 \mathrm{a}, \mathrm{b}$ shows the co-polar and cross-polar radiations patterns, respectively. In these figures, solid and dashed lines with the same color correspond to the RHCP and LHCP produced for each feed horn. Note that the six tilted main beams maintained their nice shape. For each feed horn, the desired \pm 0.28 degof tilt of the main beams were achieved with only $0.4 \mathrm{~dB}$ of loss in the gain. The cross-polar radiation was $35 \mathrm{~dB}$ below the maximum of radiation, in compliance with the typical requirements in satellite antennas for multiple spot beams in the Ka-band.

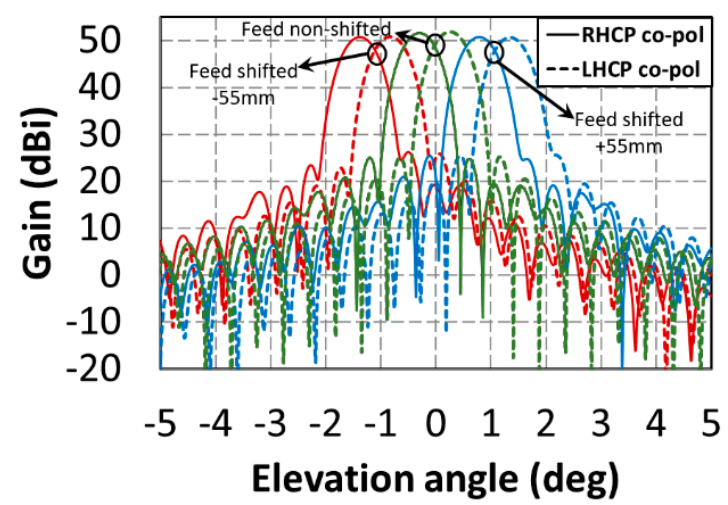

(a)

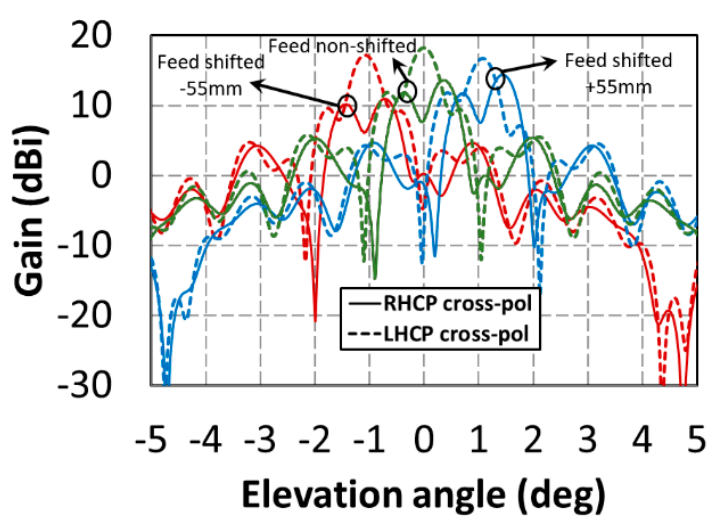

(b)

Figure 7. Elevation cuts of the (a) co-polar and (b) cross-polar radiation patterns for three positions of the feeder and for two orthogonal circular polarizations. The directions of the beams were for $\theta_{\text {beam }, 1}= \pm 0.28^{\circ}, \pm 0.83^{\circ}$, and $\pm 1.36^{\circ}$. LHCP: left-handed circular polarization.

The results shown in this section are satisfactory because they fulfill the typical stringent requirements of multi-spot beam satellite antennas $[13,14]$. In this case, two beams per feed were obtained using one aperture. According to $[13,14]$, the final challenge is to obtain four beams per feed in dual band using one aperture. This goal will employ the uses of a four-color scheme for space applications such as direct broadcast satellite service, military communication satellite, and high-speed internet applications. So, the antenna design shown in this section is a required step toward surmounting the final challenge.

\section{Conclusions}

A design technique was proposed for tilting dual CP focused beams in specular directions using VRT with rotating reflectarray elements printed on a parabolic reflector fed by a single dual CP feed horn.

A rotating reflectarray element to apply VRT made of a conductive cross embedded in a grounded multilayer substrate was proposed. The reflectarray element was optimized to provide few calls to a full-wave analysis routine in the design process, and very linear phase curves and low cross-polarization level were obtained.

To demonstrate the multi-beam capacity, the parabolic reflectarray was illuminated with three feeds, and six main spots were obtained with angular separation less than $0.56^{\circ}$ and cross-polarization level below $35 \mathrm{~dB}$ with respect to the maximum of radiation.

Author Contributions: Conceptualization, F.C.; methodology, F.C.; software, Á.S. and I.G.; validation, Á.S., R.F., and I.G.; formal analysis, F.C.; writing—original draft preparation, Á.S.; writing—review and editing, R.F.; visualization, R.F.; supervision, F.C. and I.G.; project administration, F.C.; funding acquisition, F.C. 
Funding: This research was funded in part by the Spanish Ministry of Economy and Competitiveness, Projects Ref. TEC 2017-89456-R with the support of FEDER funds, TEC2016-75103-C2-1-R and DI-14-06818, and by the European Space Agency (ESA) under contract 4000117113/ 16/ NL/AF.

Conflicts of Interest: The authors declare no conflict of interest.

\section{References}

1. Alsath, M.G.N.; Arun, H.; Selvam, Y.P.; Kanagasabai, M.; Kingsly, S.; Subbaraj, S.; Sivasamy, R.; Palaniswamy, S.K.; Natarajan, R. An integrated tri-band/UWB polarization diversity antenna for vehicular networks. IEEE Trans. Veh. Technol. 2018, 67, 5613-5620. [CrossRef]

2. Alibakhshi-Kenari, M.; Naser-Moghadasi, M.; Sadeghzadeh, R.A.; Virdee, B.S.; Limiti, E. Periodic Array of Complementary Artificial Magnetic Conductor Metamaterials-Based Multiband Antennas for Broadband Wireless Transceivers. IET Microwaves Antennas Propag. 2016, 10, 1682-1691. [CrossRef]

3. Alibakhshi-Kenari, M.; Naser-Moghadasi, M.; Sadeghzadeh, R.A.; Virdee, B.S.; Limiti, E. Bandwidth Extension of Planar Antennas Using Embedded Slits for Reliable Multiband RF Communications. AEU Int. J. Electron. Commun. 2016, 70,910-919. [CrossRef]

4. Sadeghzadeh, R.A.; Alibakhshi-Kenari, M.; Naser-Moghadasi, M. UWB Antenna Based on SCRLH-TLs for Portable Wireless Devices. Microwave Opt. Technol. Lett. 2016, 58, 69-71. [CrossRef]

5. Alibakhshi-Kenari, M.; Movahhedi, M.; Naderian, H. A New Miniature Ultra Wide Band Planar Microstrip Antenna Based on the Metamaterial Transmission Line. In Proceedings of the 2012 IEEE Asia- Pacific Conference on Applied Electromagnetics (APACE 2012), Melaka, Malaysia, 11-13 December 2012; pp. $293-297$.

6. Wu, Q.; Zhou, Y.; Guo, S. An l-sleeve 1-monopole antenna fitting a shark-fin module for vehicular LTE WLAN and car-to-car communications. IEEE Trans. Veh. Technol. 2018, 67, 7170-7180. [CrossRef]

7. Chaloun, T.; Ziegler, V.; Menzel, W. Design of a dual-polarized stacked patch antenna for wide-angle scanning reflectarrays. IEEE Trans. Antennas Propag. 2016, 64, 3380-3390. [CrossRef]

8. Li, M.; Xiao, S.Q.; Wang, B.Z. Investigation of using high impedance surfaces for wide-angle scanning arrays. IEEE Trans. Antennas Propag. 2015, 63, 2895-2901. [CrossRef]

9. Luo, Q.; Gao, S.; Li, W.; Sobhy, M.; Bakaimi, I.; Kees de Groot, C.H.; Hayden, B.; Reaney, I.; Yang, X. Multibeam Dual-Circularly Polarized Reflectarray for Connected and Autonomous Vehicles. IEEE Trans. Veh. Technol. 2019, 68, 3574-3585. [CrossRef]

10. Wen, Y.Q.; Wang, B.Z.; Ding, X. Wide-beam circularly-polarized microstrip magnetic-electric dipole antenna for wide-angle scanning phased array. IEEE Antennas Wirel. Propag. Lett. 2017, 16, 428-431. [CrossRef]

11. Luo, Q.; Gao, S.; Zhang, C.; Zhou, D.; Chaloun, T.; Menzel, W.; Ziegler, V.; Sobhy, M. Design and analysis of a reflectarray using slot antenna elements for ka-band SatCom. IEEE Trans. Antennas Propag. 2015, 63, 1365-1374. [CrossRef]

12. Varum, T.; Matos, J.N.; Pinho, P.; Abreu, R. Nonuniform broadband circularly polarized antenna array for vehicular communications. IEEE Trans. Veh. Technol. Vol. 2016, 65, 7219-7227. [CrossRef]

13. Rao, S.K. Parametric design and analysis of multiple-beam reflector antennas for satellite communications. IEEE Antennas Propag. Mag. 2003, 45, 26-34. [CrossRef]

14. Rao, S.K.; Tang, M.Q. Stepped-reflector antenna for dual-band multiple beam satellite communications payloads. IEEE Trans. Antennas Propag. 2006, 54, 801-811. [CrossRef]

15. Zhou, M.; Sorensen, S.B. Multi-Spot Beam Reflectarrays for Satellite Telecommunication Applications in Ka-Band. In Proceedings of the 2016 10th European Conference on Antennas and Propagation (EuCAP), Davos, Switzerland, 10-15 April 2016; pp. 1-5. [CrossRef]

16. Sanz-Fernández, J.; Saenz, E.; de Maagt, P. A circular polarization selective surface for space applications. IEEE Trans. Antennas Propag. 2015, 63, 2460-2470. [CrossRef]

17. Martinez-de-Rioja, E.; Encinar, J.A.; Florencio, R.; Boix, R.R. Reflectarray in K and Ka Bands with Independent Beams in Each Polarization. In Proceedings of the Reflectarray in K and Ka Bands with Independent Beams in Each Polarization, Fajardo, Puerto Rico, 26 June-1 July 2016; pp. 1199-1200. [CrossRef]

18. Smith, T.; Gothelf, U.V.; Kim, O.S.; Breinbjerg, O. Design, manufacturing, and testing of a 20/30 GHz dual-band circularly polarized reflectarray antenna in submission. IEEE Antennas Wireless Propag. Lett. 2013, 12, 1480-1483. [CrossRef] 
19. Huang, J.; Pogorzelski, R.J. A Ka-band microstrip reflectarray with elements having variable rotation angles. IEEE Trans. Antennas Propag. 1998, 46, 650-656. [CrossRef]

20. Huang, J.; Encinar, J.A. Reflectarray Antennas; IEEE Press: Piscataway, NJ, USA, 2008.

21. newFASANT Suite. Available online: www.fasant.com (accessed on 29 June 2019).

22. Somolinos, A.; Florencio, R.; González, I.; Encinar, J.A.; Cátedra, F.M. Experimental Validation of Generating Two Spaced Beams with Reflectarrays by VRT". IEEE Trans. Antennas Propag. 2019, 67, 4263-4268. [CrossRef]

23. Martynyuk, A.E.; Martinez, J.I.; Martynyuk, N.A. Spiraphase-Type Reflectarrays Based on Loaded Ring Slot Resonators. IEEE Trans. Antennas Propag. 2004, 52, 142-153. [CrossRef]

24. Eibert, T.F.; Volakis, J.L. Antenna Engineering Handbook; McGraw-Hill: Piscataway, NY, USA, 2007.

(C) 2019 by the authors. Licensee MDPI, Basel, Switzerland. This article is an open access article distributed under the terms and conditions of the Creative Commons Attribution (CC BY) license (http://creativecommons.org/licenses/by/4.0/). 